\title{
Artificial Intelligence in Health, Human Service Delivery and Education: A Brief Conceptual Overview
}

\author{
Randy Basham \\ The University of Texas at Arlington, Arlington, TX 76019, USA
}

\begin{abstract}
This paper will articulate a conceptual overview of online content currently available to assess the development of AI to improve human service delivery and social services as well as to enhance and improve educational service delivery and human condition outcomes. Artificial intelligence has been the subject of substantial optimism and being of service to improving human lifestyles. AI has become an integral aspect of the technology industry and is affecting information science transportation economic and manufacturing services. However, there are emerging applications of AI in the improvement of human welfare, health, and educational attainment. Each of these will be addressed in the conceptual review to follow.
\end{abstract}

Key words: Artificial intelligence, health, education, human service delivery.

\section{Introduction}

Artificial intelligence may be thought of as the intelligence like qualities exhibited by computing machines or software. Artificial intelligence, or AI, is also an academic field of study. Artificial intelligence attempts to render aspects of humanlike intelligence from machinery or computing devices. AI is a highly technical and specialized area of research divided into subfields that are not well integrated. The AI field of study and design is concerned with the development of intelligence agents within a system that perceives its environment and takes action and that maximizes its chances of succeeding. AI may be defined as the science and engineering of making intelligent machines.

AI research is divided into disparate and at times competing subfields of knowledge oriented toward solving a particular problem or developing a particular tool or application. These subfields are divided in part due to various social and cultural factors related to the application and its consequences on human

Corresponding author: Randy Basham, Ph.D., associate professor, research fields: emerging technology and improving the human condition. communities. Additionally, some AI research is specific to unique institutions within government, industrial, and military organizations, of which a number of applications have direct relevance for improvement of other nonrelated human problems.

The core problems or solutions sought by AI researchers include machine reasoning, knowledge acquisition, machine planning machine learning and natural process processing or communication as well as machine perception and the ability to move and manipulate objects. The acquisition of general intelligence or independent intelligence remains among the longer-term goals of scientists and researchers concerned with some popular approaches include statistical methods computational intelligence and traditional symbolic space AI, more recently has become the interest of a number of interdisciplinary fields which a number of sciences and professions converge these have included computing science psychology linguistics education and neuroscience but more recently have included human knowledge acquisition and education and improving the capacity to deliver human services efficiently.

Much of recent news media coverage on artificial intelligence has been concerned with AI capacity to 
improve rapid manufacturing and distribution techniques, four military and intelligence gathering applications, and in emergent concerns of artificial intelligence to supersede human intelligence in aggregate. AI is presumed to have the capacity for rapid and dramatic improvement of human health and the human condition and also contains the inherent risk of machine domination or eradication of the human species.

This paper will articulate a conceptual review of online content currently available to assess the development of AI to improve health and human service delivery and social services as well as to enhance and improve educational service delivery and human condition outcomes. Artificial intelligence has been the subject of substantial optimism and being of service to improving human lifestyles. AI has become an integral aspect of the technology industry and is affecting information science transportation economic and manufacturing services. However, there are emerging applications of AI in the improvement of human welfare, health, and educational attainment. Each of these will be addressed in the literature review to follow.

\section{Conceptual Overview}

The history of artificial intelligence is synonymous with the history of the development of complex machines to provide narrative storytelling or computation and reasoning to resolve problems. Much of the more recent conceptualization of artificial intelligence derives from the work of Alan Turing who conceived that by reset shuffling symbols as simple as zero and one that machines could learn to problem solve and deduce the solution to nearly any problem. The understanding that digital computers can simulate any process associated with formal reasoning has become known as the church-Turing thesis. Additional advances in neurology neurobiology information processing and cybernetics led researchers to consider the possibility of building an electronic brain.
The founders of modern AI research emerged from a workshop held at Dartmouth College in 1956. Scientists and their students developed simple computers to learn gaming strategies and eventually to play better than human beings. These advances were followed by advances in solving word problems with machine learning and proving theorems of logic. Machines thought to be capable of learning any task that humans could accomplish and doing any work that humans could do within the following generation [1].

\subsection{Gradual Advances in Artificial Intelligence}

Though additional machine learning tasks have proven difficult to develop, AI eventually began to be used for logistics and data mining and indeed pattern detection within complex sets of data. Deep learning tasks followed and were developed to include advances in machine learning and self-perception advanced algorithms are now being used to sort communication messages complete competing complex gaming and provide affordable neural networks. AI has begun to resolve problems and reasoning and problem-solving knowledge representation planning learning natural language processing perception motion a manipulation social intelligence creativity and general intelligence such that $\mathrm{AI}$ is creating tautologies allowing computers machines to function in an intelligent and an anticipated eventual independent manner [1].

\subsection{Machine Learning and Information Technology}

Artificial intelligence and machine learning have become heavily infused with the utilization of information technology. Machine learning permits automated text classification, which permits rapid search and location of terms documents and concepts. In addition, information technologies are enhanced with respect to information retrieval and the development of neural networks to organize and analyze digital content. Data mining and classification and clustering of data through artificial intelligence 
applications permit rapid pattern recognition, and assistance for human interpretation of massive data sources [2].

A number of computing and machine learning enhancements allow data to be reorganized and graphically represented such that information can be visualized for better understanding and utilization. Further, machine learning can be organized to classify concepts and sword very ideas into hierarchies. Data or materials can be distributed optimally and various sorts of statistical learning improves analysis and process control [2].

Artificial intelligence is capable of conducting materials ideas and content into various decision trees. These decision trees enhance capacity for human performance and overcoming tedium and fatigue in organizing and processing materials and information. Interestingly, as a safeguard for crisis intervention and protection of organizational and personal information, machine learning is increasingly utilized in computer intrusion detection. Moreover, relative to information technology, artificial intelligence is capable of performing computational thinking and self-correcting or self-evaluation of its own performance [2].

\subsection{Machine Learning and Human Service Automation}

One mechanism, which is at the early development phase of health and human service delivery involving machine learning, is the machines capacity within human services to automate systems and provide knowledge sharing. Interaction with remote services across prior language barriers and access issues to provide text based and wireless data has and will continue to improve access to democratic processes scientific and procedural information government communication and essential information sharing services. AI may continue to improve on this development by deploying complex algorithms to determine best fit information needs for receiver organizations and those having information to share.
Machine learning is anticipated to rapidly overtake the speed of human decision-making in providing essential information to those most in need [3].

In addition, by utilizing AI to coordinate product manufacture and transportation essential devices and products may be custom created as need or anticipated needs arises among fragile groups, and resource individual parties, and oppressed or marginalized communities. Machine learning has advanced to a state right human-based manufacturing and distribution services may be replaced with machine dominant systems to duplicate every human input into the creation of a product through traditional manufacturing, or more unique products through the advent of 3-D printing with these being delivered by way of an emerging transportation revolution directly interfaced with machine learning systems [3].

Agriculture and human food source production is also becoming heavily augmented by both machine supervised agricultural methods and computing supported bio-products manufacture and distribution. Matching human need and product or service delivery would require some tracking of the recipient population are persons. However, AI is fully capable of improving distribution products in addition to health and human service delivery and information access. Human capacity or responders may also be more rapidly deployed and do not and more exacting specialization through machine learning directives [3].

\subsection{Machine Learning and Human Betterment}

Recently, scholars have postulated that artificial intelligence could be utilized for human and societal betterment. Machine learning or artificial intelligence applications are capable of sampling the environment and determining schedules to perform ecological testing to prevent harm to children and vulnerable adults. Such tests could include building paints, architectural standards, water purification and the potential for exposure to other harmful elements. Public health inspectors could address health hazards 
and risks to communities more efficiently and more rapidly. Selected medical records could be scanned to identify pregnancies or children at risk within local environments who may or may not be offered additional testing or treatment [4].

Artificial intelligence could readily be used to track at risk youth having a high likelihood of school dropout. Social factors, employment factors, economic factors, school performance issues, and juvenile databases relative to crime could be accessed to determine the risk factor of non-completion of school for any particular student. Additional advising, tutoring, community programs and social and familial supports could be provided to assure success.

Public safety could potentially be enhanced through identification of public service servants to include the police who may be at risk of performance issues within their career such as excessive violence, absenteeism, and adequate past performance, inadequate training schedules, and records of incidents that led to administrative review. Additional supports could be provided to include paid leave her training leave to improve performance and reduce risk [4].

There is the need to develop data infrastructure and data platforms of which human services decision-makers public service providers could identify at risk individuals and populations. Such datasets might include healthcare data educational data criminal records public servant job performance records and even economic or tax data.

Community betterment could include such things as economic development. Home and building inspections or small business inspections could occur with more frequency if the process and scheduling accelerated with the aid of artificial intelligence. Areas needing infrastructure repairs, or maintenance, could be identified more quickly and prevent failed infrastructure issues. With sufficient effort, urban blight could be reduced and neighborhoods and communities maintained prosperously and safely [4].

There are a number of possible ways that artificial intelligence could be deployed to improve social networks. Health and human service delivery entities could benefit from improved data collection and maintenance and analysis around community priorities. Data sources could be integrated such that they work in combination to provide more accurate assessments of community problem areas and prevent negative outcomes. Machine learning statistical and predictive models of group and individual behaviors could be enhanced to improve community interventions. Public policies could be assessed over time for effectiveness and modified upgraded or abolished based upon their data-driven evidence of effectiveness [4].

\subsection{Machine Learning and the Reduction of Poverty}

More compelling in recent years is the emerging conceptualization that artificial intelligence or machine learning can address at least partially the causes of poverty and perhaps provide some solutions for the reduction of poverty. This is, of course, a major humanistic goal for social scientists and health and human service delivery organizations. Though there is an understanding among industry leaders and government decision-makers that there is a threat of artificial intelligence leading to large-scale unemployment in some sectors of occupations, there is also a possibility of matching the unemployed to available trades and occupations or training for such.

Machine learning in combination with the earlier mentioned learning management systems could address preparation for occupations through differential education and match students individually with varying learning styles or strengths with specified occupational outcomes. Such training could reduce the costs of government services by reducing dependency on the services. Training and job matching in combination with directed artificial intelligence may serve to reduce struggling for employment and improve job placement satisfaction. Occupational, personnel, and other large data sets could examine trends in employment or expected 
trends in employment and predict future job openings sometime before their needed [5].

\subsection{Machine Learning in Education and Delivery of Education}

In terms of education, and the delivery of educational content artificial intelligence has made great strides to facilitate education within the past few years. Coding and programs have been developed to permit machine learning to better understand student patterns of performance and thereby understand the various ways of which students learn. This process becomes helpful in informing educators and educational program decision-makers via the provision of differential data sets to analyze and comprehend the educational needs of individual students and in aggregate course cohorts and classes in their entirety.

A number of learning management systems (LMS) and educational platforms for course delivery have been developed that immerse the student into the learning experience. These systems are also referred to as virtual learning environments and may use three-dimensional data processing and imaging, avatars augmented reality virtual reality and the interaction of hardware machining and robots to facilitate the educational experience. These applications are assisting with astronomical discovery, Earth ecosystem and environment discovery, raw materials identification and extraction, scientific and medical training, and various professional and technical occupations [6].

Learning management systems are more broadly used to facilitate classroom instruction and provide hybrid or blended courses which are partially face-to-face and online and for completely online educational training. These serve as a portal for students to access content relative to a course, submit assigned work and receive feedback on performance. There is controversy among educators as to whether these platforms serve the role of learning, or more simply the administration of learning content.
An emerging theme in developing advanced learning management systems is the capacity for the student learner to gain greater personalization of learning content while adhering to common learning platform design standards and to increase the role of artificial intelligence relative to formative learning and assessment. These advanced learning management systems are being designed to allow the reorganization and repurposing of learning content in an endless variety of learning opportunities. Emerging educational applications would permit unique personalization and improve engagement [6].

\subsection{Artificial Intelligence and Planned Obsolesce of the Educator}

A number of disparate and varied applications and engineering marvels have begun to merge to provide some potential for eventually taking over the development of education content, syllabi, and assessment of student competency. Coupled with advanced learning management systems in continual reduction in barriers to access of content, these innovations have emergent capacity to replace human educators entirely over time [7].

Educational content can be delivered by avatars, or Computer Generated Imaging (CGI) to provide model instructors, document readers and eventual computer rendered graphical representations and illustrations, can provide rich understanding of complex constructs. A number of fairly effective computing programs currently exist and are improving to offer online assessment, online tasting's, and even the capacity to read and grade accurately written assignments, both for content and writing style.

Segment of the educational industry itself is at risk for planned obsolesce. Improved machine learning systems, may make decisions about which content and knowledge is most useful to have available and in what formats based on computer perceived occupational or production needs, without consideration to various codes of values or ethics that 
would have apply to human delivery systems.

\subsection{The Future of Artificial Intelligence in Human Services and Education}

Artificial Intelligence or advanced applied machine learning holds promise of steady gains, in improving error reduction, and providing more reliable and efficient services in health and human service delivery and education. Though AI is not seen as a panacea to resolve all human disparities and limitations, there is substantial optimism for a more equitable distribution of goods and services, as well as, knowledge and skill development among those whom previously had limited access to education or reliable employment. There is no corresponding engineering model for resource and wealth distribution, however. The late Dr. Stephen Hawking, recently observed [8]:

"If machines produce everything we need, the outcome will depend on how things are distributed," he wrote. "Everyone can enjoy a life of luxurious leisure if the machine-produced wealth is shared, or most people can end up miserably poor if the machine-owners successfully lobby against wealth redistribution.”

\section{Discussion}

There are a number of anticipated technologies based revolutions in process and likely to occur over the next few years. Most of these will incorporate aspects of Artificial Intelligence or machine learning. Some of those expected, include rapid technological advances in manufacturing, development of stable food supplies, reductions in common illnesses, low cost and readily available transportation, home delivery of almost any needed product, more efficient and comfortable housing, and lower energy costs. Likely most of these will affect humans service delivery and education and training of rapidly changing workforces. A number of large economic benefits will likely be realized, though the equity of distribution of these benefits is less certain.
More certain though, is the probability that human services and education will be transformed to increasingly rely less on human input. Artificial Intelligence and/machine learning which only a few tears previous were thought to be far futuristic scientific and engineering goals are rapidly approaching as pragmatic realities of our time. Those key decision makers in both human services and education at all levels need to be transitioning now to meet this new service reality.

\section{References}

[1] AI Topics: An Official Publication of the AAAI. 2018. “A Brief History of AI." Retrieved from https://aitopics.org/misc/brief-history.

[2] SAS, The Power to Know. 2018. "Artificial Intelligence and Why It Matters.” Retrieved from https://www.sas.com/en_us/insights/analytics/what-is-arti ficial-intelligence.html.

[3] Fishman, T. D., Eggers, W. D., and Kishnani, P. 2017. "AI-Augmented Human Services Using Cognitive Technologies to Transform Program Delivery.” Retrieved from

https://www2.deloitte.com/insights/us/en/industry/publicsector/artificial-intelligence-technologies-human-services -programs.html (October 2017).

[4] Wei, A., and Baofeng, Z. 2016. "Using AI for the Betterment of Humanity: Keeping It Real with Educated AI, Huawei 2012 Labs.” Retrieved from http://www.huawei.com/en/about-huawei/publications/wi nwin-magazine/AI/using-ai-for-betterment-of-humanity.

[5] Mason, E. 2018. "AI and Big Data Could Power a New War on Poverty." Retrieved from https://www.straitstimes.com/opinion/ai-and-big-data-cou ld-power-a-new-war-on-poverty (January 2018).

[6] New Media Consortium. 2017. "NMC Horizon Report: 2017 Higher Education Edition.” Retrieved from https://www.nmc.org/publication/nmc-horizon-report-201 7-higher-education-edition/.

[7] Barnwell, P. 2017. “Are Teachers Becoming Obsolete?” Retrieved from https://www.theatlantic.com/education/archive/2017/02/b ecoming-obsolete/516732/ (February 2017).

[8] Grossman, S. 2015. "Stephen Hawking Revealed Life's Most Intriguing Mystery in HisReddit AMA.” Retrieved from http://time.com/4066421/stephen-hawking-reddit-ama/ (October 2015). 